\title{
Methylmalonic Acid and Homocysteine as Indicators of Vitamin B12 Deficiency in Patients with Gastric Cancer after Gastrectomy
}

\author{
Sae-Mi Lee ${ }^{1,2}$, Jongwon $\mathrm{Oh}^{2}$, Mi-Ryung Chun ${ }^{2} \mathbb{1}$ and Soo-Youn Lee ${ }^{2, *(D)}$ \\ 1 GC genome, 107, Ihyeon-ro 30beon-gil, Giheung-gu, Yongin-si, Gyeonggi-do 16924, Korea; \\ saemi.lee@greencross.com \\ 2 Department of Laboratory Medicine and Genetics, Samsung Medical Center, Sungkyunkwan University \\ School of Medicine, 81 Irwon-ro, Gangnam-gu, Seoul 06351, Korea; jongwon1234.oh@samsung.com (J.O.); \\ miryung.chun@samsung.com (M.-R.C.) \\ * Correspondence: suddenbz@skku.edu; Tel.: +82-2-3410-1834; Fax: +82-2-3410-2719
}

Received: 8 February 2019; Accepted: 19 February 2019; Published: 21 February 2019

\begin{abstract}
Vitamin B12 deficiency is a common complication in patients after gastrectomy. Elevated methylmalonic acid (MMA) and homocysteine are better indications of vitamin B12 deficiency than vitamin B12 serum level. We compared MMA and homocysteine levels of patients with gastric cancer after gastrectomy $(n=151)$ with controls $(n=142)$ and evaluated the prevalence of vitamin B12 deficiency using MMA and homocysteine in patients. MMA and homocysteine levels were significantly higher $(p<0.05)$ in patients with gastric cancer after gastrectomy. Of the 151 patients assessed after gastrectomy, 32 patients $(21.2 \%)$ were vitamin B12 deficient as defined by serum MMA levels $>350 \mathrm{nmol} / \mathrm{L}$, and 8 patients (5.3\%) were vitamin B12 deficient as defined by serum homocysteine levels $>15 \mu \mathrm{mol} / \mathrm{L}$. Both MMA and homocysteine levels were elevated in 7 patients. Among 33 patients with elevated MMA or homocysteine levels, 8 patients $(24.2 \%)$ were vitamin B12 deficient based on a serum vitamin B12 level $<200 \mathrm{pg} / \mathrm{mL}$. Additionally, levels of MMA and homocysteine were compared pre- and post-gastrectomy in 27 patients. The median MMA level was higher in patients with post-gastrectomy compared to pre-gastrectomy, while the median serum homocysteine level was not significantly different. These results indicate that using serum vitamin B12 levels alone may fail to detect vitamin B12 deficiency. Additional assessments of MMA and homocysteine levels are useful to evaluate possible vitamin B12 deficiency in patients who underwent a gastrectomy, and MMA is a better indicator than homocysteine to detect early changes in vitamin B12 levels.
\end{abstract}

Keywords: methylmalonic acid; homocysteine; vitamin B12; gastric cancer; gastrectomy

\section{Introduction}

Vitamin B12 is essential for DNA synthesis and maintenance of normal hematologic and neurologic functions [1]. It acts as a cofactor for two enzymatic reactions: the conversion of methylmalonic acid (MMA) to succinic acid and the synthesis of methionine from homocysteine. Acquired vitamin B12 deficiency or congenital vitamin B12 metabolism disorders impair both enzymatic reactions. Acquired vitamin B12 deficiencies are caused by inadequate dietary intake, malabsorption, or other medical conditions such as pernicious anemia [2]. A gastrectomy is a well-known cause of vitamin B12 deficiency [3]. A gastrectomy results in lack of intrinsic factors and causes gradual depletion of vitamin B12 stores, with deficiency occurring 1-2 years post-surgery $[3,4]$. Gastric cancer is a common malignancy in Korea [5], and patient survival has increased as a result of early detection and improved treatment protocols. Therefore, screening and treatment of vitamin 
B12 deficiency have become more important for improving the quality of life in gastric cancer patients [6]. The serum vitamin B12 assay currently used as the initial routine diagnostic test has several limitations including poor agreement between laboratories, methods, or platforms and low sensitivity and specificity $[4,7]$. To overcome these limitations, several markers associated with vitamin B12 metabolism could be used in place of a single vitamin B12 assay. MMA and homocysteine, which accumulate in the absence of adequate vitamin B12, are sensitive and specific markers of vitamin B12 function and are used to diagnose vitamin B12 deficiency at an early stage and monitor treatment effects [8]. Vitamin B12 deficiency may be underestimated in patients with gastric cancer who underwent gastrectomy when assessed using measurements of vitamin B12 levels because serum vitamin B12 levels do not reflect functional vitamin B12 deficiency.

MMA and homocysteine levels have been evaluated as early markers of vitamin B12 deficiency in different groups including the general population, elderly, infants, pregnant women, and healthy women [7,9-13]. However, studies measuring both MMA and homocysteine in patients who underwent gastrectomy are limited. Therefore, in this study, we compared MMA and homocysteine levels in patients after gastrectomy with healthy controls and determined the prevalence of vitamin B12 deficiency using MMA and homocysteine levels in patients after gastrectomy. We also compared MMA and homocysteine levels in patients before and after gastrectomy.

\section{Materials and Methods}

\subsection{Study Population}

This study enrolled 151 Korean adult patients with gastric cancer who underwent gastrectomy and were followed up at Samsung Medical Center (Seoul, Korea), a tertiary care hospital. Individuals for the control group were selected from adults without any clinical symptoms or signs of illness that had visited the health promotion center for a medical checkup. Inclusion criteria for the control group were as follows: (1) No medical history of diseases such as malignancy, renal and thyroid dysfunction, psoriasis, and diabetes, and (2) no history of certain medications that have been associated with elevated homocysteine levels (anticonvulsant agents, folate antagonists, cholestyramine, metformin, thiazide diuretics, cyclosporine, levodopa). Demographic data including age, sex, operation date, and medications prescribed were obtained from electronic medical records. Hematologic and biochemical parameters including hemoglobin $(\mathrm{Hb})$, mean corpuscular volume $(\mathrm{MCV})$, creatinine, serum total protein, albumin, aspartate aminotransferase (AST), and alanine aminotransferase (ALT) were measured concurrently with MMA and homocysteine. The following levels were used to define vitamin B12 deficiency based on previously published research: (1) MMA level > $350 \mathrm{nmol} / \mathrm{L}$ [14], (2) homocysteine level $>15 \mu \mathrm{mol} / \mathrm{L}$ [15], and (3) serum vitamin B12 level $<200 \mathrm{pg} / \mathrm{mL}$. Among patients who underwent gastrectomy, vitamin B12 was also measured in patients whose MMA or homocysteine levels were above the defined deficiency level. In addition, MMA and homocysteine were measured before and after gastrectomy in 27 patients who survived for more than five years after gastrectomy. Among them, 6 patients underwent total gastrectomy and 21 patients underwent subtotal gastrectomy. Of 6 patients who underwent total gastrectomy, 5 were treated with an intramuscular injection of cobamamide (1000 $\mu$ g once a month to once a year, varied by patients) and 1 was treated with oral administration of cyanocobalamin after gastrectomy $(0.25 \mathrm{mg}$ twice a day). While, 27 patients who underwent subtotal gastrectomy were not treated. This study was approved by the Institutional Review Board of Samsung Medical Center (IRB No. SMC 2015-07-027-006) and written consents were obtained for subjects who need additional blood collection.

\subsection{Analytical Procedures}

Blood samples were collected in red stoppered plain tubes (Becton Dickinson Co., Franklin Lakes, NJ, USA). Serum was separated from whole blood and immediately stored at $-20{ }^{\circ} \mathrm{C}$ until assayed. Serum MMA and homocysteine levels were measured on an Acquity ultra-performance 
liquid chromatography (UPLC) (Waters Corporation, Milford, MA, USA) connected to a Xevo TQ-S tandem mass spectrometer (Waters Corporation, Milford, MA, USA) using the modified method described by Hempen et al. [16]. Intra- and inter-assay imprecision were both $<10 \%$ of the coefficient of variation. The accuracy of the homocysteine assay was ensured using the Proficiency Testing/Quality Management program of the United States College of American Pathologists survey. The assay detection ranges were 45.0-2810.0 nmol/L for MMA and 5.0-60.0 $\mu \mathrm{mol} / \mathrm{L}$ for homocysteine.

Serum vitamin B12 levels were measured with a chemiluminescent immunoassay using an ADIVA Centaur XP (Siemens Diagnostics, NY, USA). Hb and MCV were analyzed using Sysmex XE-2100 and XN-9000 (Sysmex, Kobe, Japan) hematology analyzers, and creatinine, serum total protein, albumin, AST, and ALT levels were measured using a Roche modular analyzer (Roche Diagnostics Corp., Indianapolis, IN, USA) according to the manufacturer's instructions.

\subsection{Statistical Analysis}

Data were analyzed using SPSS software v23.0 (SPSS Inc. 233 S. Chicago, IL, USA). The independent sample t-test and Wilcoxon rank sum test were used to assess the significant differences between gastrectomy patients and controls for continuous values. Categorical variables were compared using the Chi-square test. To assess differences in MMA and homocysteine levels measured before and after gastrectomy, the Wilcoxon signed rank test was used. $p$-values less than 0.05 were considered statistically significant.

\section{Results}

This study included 151 patients that had a previous gastrectomy (87 male and 64 female) and 142 healthy controls ( 87 male and 55 female). The median follow-up period was 35 months with a range of 1-166 months. The demographic characteristics, hematologic, and biochemical results of patients and controls are shown in Table 1. Age, $\mathrm{Hb}, \mathrm{MCV}$, albumin, and total cholesterol were significantly higher in the control group. There were no significant differences between gastrectomy patient and healthy control groups in sex, creatinine, total protein, AST, and ALT.

Table 1. Comparison of demographic characteristics and biochemical markers between the control group $(n=142)$ and the gastrectomy patient group $(n=151)$.

\begin{tabular}{cccc}
\hline Parameter & Controls $(\boldsymbol{n = 1 4 2 )}$ & $\begin{array}{c}\text { Gastrectomy } \\
\text { Patients }(\boldsymbol{n}=\mathbf{1 5 1})\end{array}$ & $p$-Value \\
\hline Age (years) & $52(33-75)$ & $56(32-78)$ & $0.001^{\mathrm{a}}$ \\
Sex & & & $0.525^{\mathrm{b}}$ \\
Male (n) & 87 & 87 & \\
Female (n) & 55 & 64 & $<0.001^{\mathrm{c}}$ \\
Hemoglobin (g/dL) & $14.5(1.3)$ & $12.4(1.9)$ & $<0.001^{\mathrm{a}}$ \\
MCV (fL) & $92.5(85.3-102.9)$ & $90.3(63.3-111.0)$ & $0.159^{\mathrm{a}}$ \\
Creatinine (mg/dL) & $0.84(0.55-0.97)$ & $0.84(0.55-1.42)$ & $0.568^{\mathrm{a}}$ \\
Total protein (g/dL) & $7.0(6.3-7.9)$ & $7.0(3.8-8.2)$ & $<0.001^{\mathrm{a}}$ \\
Albumin $(\mathrm{g} / \mathrm{dL})$ & $4.6(4.1-5.1)$ & $4.3(2.5-5.0)$ & $<0.001^{\mathrm{c}}$ \\
Total cholesterol (mg/dL) & $195.9(32.8)$ & $168.3(33.1)$ & $0.099^{\mathrm{a}}$ \\
AST (U/L) & $23(12-81)$ & $24(13-123)$ & $0.115^{\mathrm{a}}$ \\
ALT (U/L) & $21(7-97)$ & $20(7-93)$ & $<0.001^{\mathrm{a}}$ \\
MMA (nmol/L) & $125.7(48.4-291.5)$ & $191.3(60.2-1374.6)$ & $0.044^{\mathrm{a}}$
\end{tabular}

Abbreviations: ALT, alanine aminotransferase; AST, aspartate aminotransferase; MCV, mean corpuscular volume; MMA, methylmalonic acid. a results are presented as median (range) with $p$-values from a Wilcoxon rank sum test; ${ }^{\mathrm{b}} p$-values from the chi-square test; ${ }^{\mathrm{c}}$ results are presented as mean (standard deviation) with $p$-values from student t-test.

The median serum MMA level was significantly higher in gastrectomy patients than in controls (191.3 vs. $125.7 \mathrm{nmol} / \mathrm{L}, p<0.001)$, and the median serum homocysteine level was also significantly higher in gastrectomy patients (10.0 vs. $9.5 \mu \mathrm{mol} / \mathrm{L}, p=0.044)$ (Figure 1). Additionally, we compared 109 gastrectomy patients and 134 controls below 65 years old. There were no significant differences in 
age (53 vs. $52, p=0.891)$. The median MMA level was higher for patients after gastrectomy (188.3 vs. $124.3 \mathrm{nmol} / \mathrm{L}, p<0.001)$, but the median serum homocysteine level was not significantly different $(9.4$ vs. $9.5, p=0.804)$.

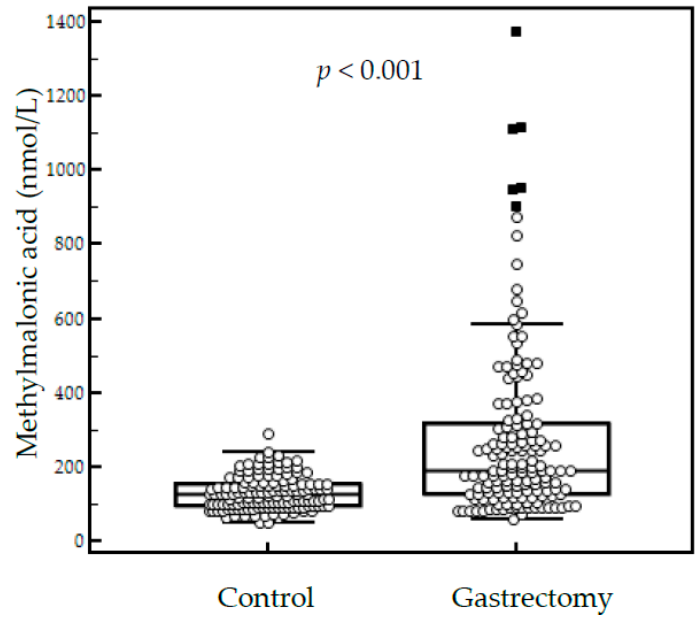

(a)

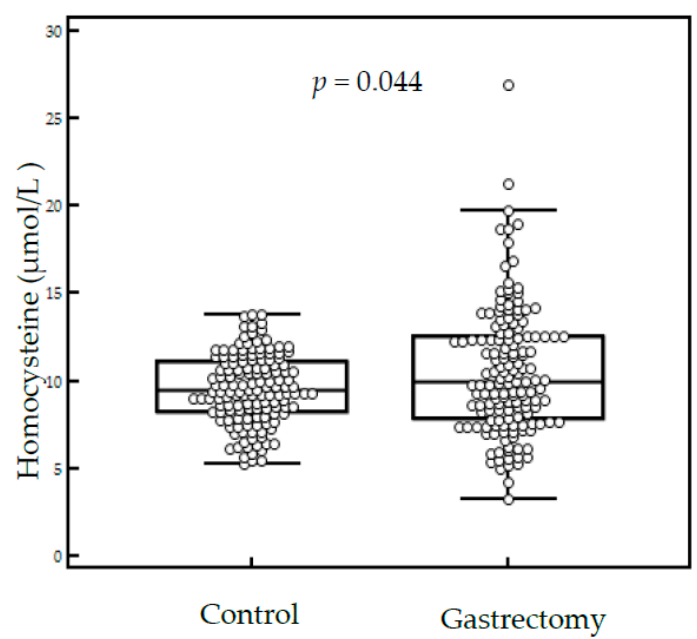

(b)

Figure 1. Serum methylmalonic acid (a) and homocysteine levels (b) in the control group $(n=142)$ and gastrectomy patient group $(n=151)$.

Of the 151 gastrectomy patients, 32 patients (21.2\%) were vitamin B12 deficient based on serum MMA levels $>350 \mathrm{nmol} / \mathrm{L}$, and eight patients (5.3\%) were vitamin B12 deficient based on serum homocysteine levels $>15 \mu \mathrm{mol} / \mathrm{L}$. MMA and homocysteine were both elevated in seven patients. Among the 33 patients with elevated MMA or homocysteine levels, eight patients (24.2\%) had low serum vitamin B12 levels. Therefore, using MMA and homocysteine as indicators of vitamin B12 deficiency detects more vitamin B12-deficient patients. No individuals in the healthy control group had elevated MMA or homocysteine levels.

In 27 patients who survived for more than five years after gastrectomy, the median MMA level was higher in patients after gastrectomy compared to before a gastrectomy $(163.8 \mathrm{vs} .116 .2 \mathrm{nmol} / \mathrm{L}$, $p<0.001)$. However, serum homocysteine levels were not significantly different after gastrectomy (10.5 vs. 10.6, $p=0.708$ ) (Figure 2). Although no individuals had elevated MMA after gastrectomy, an increase was observed comparing MMA levels pre- and post-gastrectomy.

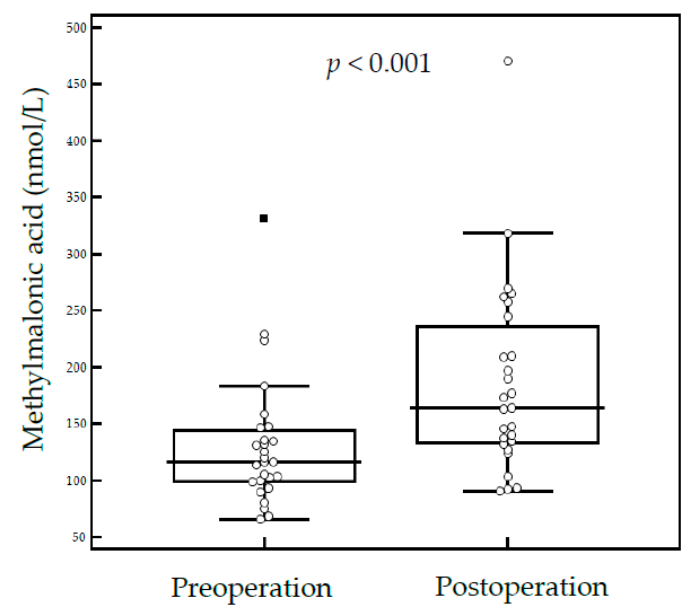

(a)

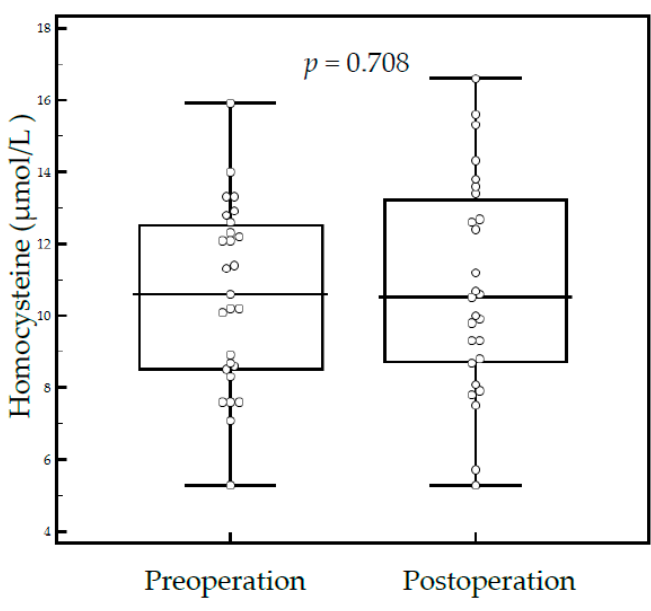

(b)

Figure 2. Serum methylmalonic acid (a) and homocysteine levels (b) in patients before and after gastrectomy $(n=27)$. 


\section{Discussion}

Early detection of vitamin B12 deficiency is clinically important because the neurologic symptoms associated with vitamin B12 deficiency cannot be reversed and pernicious anemia will occur if diagnosed late but can be prevented with a vitamin B12 supplement $[1,17]$. There is no gold standard for diagnosing vitamin B12 deficiency. Due to limitations of assays that directly measure vitamin B12, metabolites that accumulate as a result of vitamin B12 deficiency are now measured in clinical settings [18].

In this study, we determined the prevalence of vitamin B12 deficiency using MMA and homocysteine levels. The prevalence of vitamin B12 deficiency was 21.9\% (33/151) of gastrectomy patients as defined by elevated MMA or homocysteine levels. Of those patients, only 24.2\% (8/33) were vitamin B12 deficient based on serum vitamin B12 levels $<200 \mathrm{pg} / \mathrm{mL}$. Therefore, by measuring MMA and homocysteine levels, we found vitamin B12 deficiencies that were not detected by measuring vitamin B12 alone. These findings suggest that only measuring vitamin B12 could miss vitamin B12 deficiency in some patients.

We also evaluated the utility of MMA and homocysteine as follow-up markers after gastrectomy by comparing MMA and homocysteine levels in patients before and after gastrectomy. Although a small number of patients were included, there was a significant increase in MMA levels after gastrectomy. However, homocysteine was not significantly increased post-gastrectomy compared to pre-gastrectomy. This suggests that MMA could be a better follow-up marker than homocysteine in patients after gastrectomy. The superiority of MMA sensitivity to that of homocysteine is well known. An elevated MMA level is more sensitive and specific for vitamin B12 deficiency than an elevated homocysteine level because homocysteine is also increased in folate deficiency, vitamin B6 deficiency and other conditions such as renal failure and hypothyroidism [19,20].

It was reported that a vitamin B12 deficiency prevalence was 55.6\% (40/72 patients) at a median follow-up of 29 months after gastrectomy based on vitamin B12 levels $<200 \mathrm{pg} / \mathrm{mL}$ [21]. In this study, homocysteine was significantly elevated in patients after gastrectomy compared to healthy controls (15.1 vs. $12.5 \mu \mathrm{mol} / \mathrm{L}, p=0.016)$. Another study reported a vitamin B12 deficiency prevalence of $28.5 \%$ (184/645 patients) at a median follow-up of 24 months in patients after gastrectomy based on vitamin B12 levels $<200 \mathrm{pg} / \mathrm{mL}$ [22]. They report that homocysteine was significantly elevated in the vitamin B12 deficient group compared to the vitamin B12 non-deficient group (12.9 vs. $12.0 \mu \mathrm{mol} / \mathrm{L}$, $p=0.006)$. Sakuta et al. reported homocysteine was higher in gastrectomy patients than in controls (11.7 vs. $9.3 \mu \mathrm{mol} / \mathrm{L}, p=0.011$ ) [23]. In our study, the prevalence of vitamin B12 deficiency was $21.9 \%$ (33/151 patients) based on elevated MMA or homocysteine levels at a median follow-up of 35 months after gastrectomy. MMA and homocysteine were significantly elevated in gastrectomy patients compared to healthy controls (MMA: 191.3 vs. $125.7 \mathrm{nmol} / \mathrm{L}, p<0.001$; homocysteine: 10.0 vs. $9.5 \mu \mathrm{mol} / \mathrm{L}, p=0.044)$. These studies reported that homocysteine was elevated in gastrectomy patients compared to control groups, which is in accordance with the results of this study. But we could not rule out the effect of older age in gastrectomy patients group. Subgroup analysis in 109 gastrectomy patients and 134 controls below 65 years old, homocysteine level was not significantly different. This result may support the low sensitivity of homocysteine.

The prevalence of vitamin B12 deficiency varies by study because of differences in the definition of vitamin B12 deficiency and different cut-off levels, operation types, and follow-up periods. A worldwide consensus on the definition of vitamin B12 deficiency is needed to accurately evaluate the prevalence of vitamin B12 deficiency in gastrectomy patients. Using different cut-off levels to define vitamin B12 deficiency could affect the prevalence of vitamin B12 deficiency. For instance, it was reported that an MMA cut-off level of $0.376 \mathrm{nmol} / \mathrm{L}$ resulted in a prevalence of $2.4 \%$, whereas with a cut-off level of $0.271 \mathrm{nmol} / \mathrm{L}$ the prevalence was $5.9 \%$ in the general population [18]. Further studies to define standardized cut-off levels are needed. Additionally, patient composition depending on the extent of the gastrectomy could affect the prevalence of vitamin B12 deficiency. As such, in previous studies, most vitamin B12 deficiencies occurred in patients who had undergone total gastrectomy. Lim et al. reported that at 48 months after gastrectomy, the incidence of vitamin B12 deficiency was 
$3.2 \%$ in patients that had a Billroth I subtotal gastrectomy, $7.5 \%$ in patients that had a Billroth II subtotal gastrectomy, and $76.9 \%$ in patients that had total gastrectomy [24]. This study also reported that the incidences of vitamin B12 deficiency in patients who underwent total gastrectomy were $0 \%, 16.1 \%$, $50.0 \%$, and $76.9 \%$ at $12,24,36$, and 48 months after gastrectomy, respectively. Therefore, the incidence of vitamin B12 deficiency increased as time increased after gastrectomy.

Our study had several limitations. First, it was difficult to evaluate the accurate prevalence of vitamin B12 deficiency in patients with a gastrectomy due to the absence of a gold standard and various sensitivity and specificity of each diagnostic marker. High concentration of MMA or homocysteine can serve only a "possible" diagnosis of vitamin B12 deficiency. Therefore, vitamin B12 deficiency defined by MMA and homocysteine levels could detect patients with the potential for vitamin B12 deficiency. Second, it was a retrospective study and some information was unavailable. For instance, information on the use of dietary supplements, vitamins, or medications from another hospital was not collected. Third, we did not classify patients based on the extent of resection or history of chemotherapy, and the MMA and homocysteine measurements were done at various times after gastrectomy. Fourth, serum vitamin B12 levels were not measured in all patients and controls due to lack of sample volume. However, this study provides valuable information to the field because it is the first to simultaneously measure MMA and homocysteine levels in patients after gastrectomy and also the first to evaluate MMA and homocysteine as early markers of vitamin B12 deficiency in gastrectomy patients.

\section{Conclusions}

In conclusion, we simultaneously measured serum MMA and homocysteine levels and evaluated the prevalence of vitamin B12 deficiency in patients who underwent gastrectomy vs. controls. The measurement of MMA and homocysteine enabled detection of patients with possible vitamin B12 deficiency that may be missed by using the measurement of vitamin B12 alone. Further studies using various markers associated with vitamin B12 metabolism are needed to define the accurate prevalence of vitamin B12 deficiency and comprehensively and prospectively assess the utility of MMA and homocysteine as early markers of vitamin B12 deficiency in patients who underwent gastrectomy.

Author Contributions: All authors contributed to manuscript preparation; S.-M.L., J.O., M.-R.C. and S.-Y.L. collected the samples or data or contributed to data analysis; S.-M.L. and S.-Y.L. designed the study; S.-Y.L. had full access to all the data in the study and takes responsibility for the integrity of the data and the accuracy of the data analysis. All authors read and approved the final manuscript.

Funding: This research was supported by the Bio \& Medical Technology Development Program of the National Research Foundation (NRF) funded by the Ministry of Science, ICT \& Future Planning (2016M3A9B6026775).

Conflicts of Interest: The authors declare no conflict of interest.

\section{References}

1. Oh, R.; Brown, D.L. Vitamin B12 deficiency. Am. Fam. Physician 2003, 67, 979-986. [PubMed]

2. Allen, L.H. How common is vitamin B-12 deficiency? Am. J. Clin. Nutr. 2009, 89, 693S-696S. [CrossRef] [PubMed]

3. Beyan, C.; Beyan, E.; Kaptan, K.; Ifran, A.; Uzar, A.I. Post-gastrectomy anemia: Evaluation of 72 cases with post-gastrectomy anemia. Hematology 2007, 12, 81-84. [CrossRef] [PubMed]

4. Devalia, V.; Hamilton, M.S.; Molloy, A.M.; British Committee for Standards in Haematology. Guidelines for the diagnosis and treatment of cobalamin and folate disorders. Br. J. Haematol. 2014, 166, 496-513. [CrossRef] [PubMed]

5. Oh, C.M.; Won, Y.J.; Jung, K.W.; Kong, H.J.; Cho, H.; Lee, J.K.; Lee, D.H.; Lee, K.H.; Community of Population-Based Regional Cancer Registries. Cancer statistics in Korea: Incidence, mortality, survival, and prevalence in 2013. Cancer Res. Treat. 2016, 48, 436-450. [CrossRef] [PubMed]

6. Kim, H.I.; Hyung, W.J.; Song, K.J.; Choi, S.H.; Kim, C.B.; Noh, S.H. Oral vitamin B12 replacement: An effective treatment for vitamin B12 deficiency after total gastrectomy in gastric cancer patients. Ann. Surg. Oncol. 2011, 18, 3711-3717. [CrossRef] [PubMed] 
7. Bailey, R.L.; Carmel, R.; Green, R.; Pfeiffer, C.M.; Cogswell, M.E.; Osterloh, J.D.; Sempos, C.T.; Yetley, E.A. Monitoring of vitamin B-12 nutritional status in the united states by using plasma methylmalonic acid and serum vitamin B-12. Am. J. Clin. Nutr. 2011, 94, 552-561. [CrossRef] [PubMed]

8. Hoey, L.; Strain, J.J.; McNulty, H. Studies of biomarker responses to intervention with vitamin B-12: A systematic review of randomized controlled trials. Am. J. Clin. Nutr. 2009, 89, 1981S-1996S. [CrossRef] [PubMed]

9. Palacios, G.; Sola, R.; Barrios, L.; Pietrzik, K.; Castillo, M.J.; Gonzalez-Gross, M. Algorithm for the early diagnosis of vitamin B12 deficiency in elderly people. Nutr. Hosp. 2013, 28, 1447-1452. [PubMed]

10. Valente, E.; Scott, J.M.; Ueland, P.M.; Cunningham, C.; Casey, M.; Molloy, A.M. Diagnostic accuracy of holotranscobalamin, methylmalonic acid, serum cobalamin, and other indicators of tissue vitamin $\mathrm{B}_{12}$ status in the elderly. Clin. Chem. 2011, 57, 856-863. [CrossRef] [PubMed]

11. Schneede, J.; Dagnelie, P.C.; van Staveren, W.A.; Vollset, S.E.; Refsum, H.; Ueland, P.M. Methylmalonic acid and homocysteine in plasma as indicators of functional cobalamin deficiency in infants on macrobiotic diets. Pediatr. Res. 1994, 36, 194-201. [CrossRef] [PubMed]

12. Choi, R.; Choi, S.; Lim, Y.; Cho, Y.Y.; Kim, H.J.; Kim, S.W.; Chung, J.H.; Oh, S.Y.; Lee, S.Y. A prospective study on serum methylmalonic acid and homocysteine in pregnant women. Nutrients 2016, 8, 797. [CrossRef] [PubMed]

13. Schroder, T.H.; Quay, T.A.; Lamers, Y. Methylmalonic acid quantified in dried blood spots provides a precise, valid, and stable measure of functional vitamin B-12 status in healthy women. J. Nutr. 2014, 144, 1658-1663. [CrossRef] [PubMed]

14. Miller, J.W.; Garrod, M.G.; Rockwood, A.L.; Kushnir, M.M.; Allen, L.H.; Haan, M.N.; Green, R. Measurement of total vitamin B12 and holotranscobalamin, singly and in combination, in screening for metabolic vitamin B12 deficiency. Clin. Chem. 2006, 52, 278-285. [CrossRef] [PubMed]

15. Hunt, A.; Harrington, D.; Robinson, S. Vitamin B12 deficiency. BMJ 2014, 349, g5226. [CrossRef] [PubMed]

16. Hempen, C.; Wanschers, H.; van der Sluijs Veer, G. A fast liquid chromatographic tandem mass spectrometric method for the simultaneous determination of total homocysteine and methylmalonic acid. Anal. Bioanal. Chem. 2008, 391, 263-270. [CrossRef] [PubMed]

17. Lildballe, D.L.; Fedosov, S.; Sherliker, P.; Hin, H.; Clarke, R.; Nexo, E. Association of cognitive impairment with combinations of vitamin $\mathrm{B}_{12}$-related parameters. Clin. Chem. 2011, 57, 1436-1443. [CrossRef] [PubMed]

18. Yetley, E.A.; Pfeiffer, C.M.; Phinney, K.W.; Bailey, R.L.; Blackmore, S.; Bock, J.L.; Brody, L.C.; Carmel, R.; Curtin, L.R.; Durazo-Arvizu, R.A.; et al. Biomarkers of vitamin B-12 status in nhanes: A roundtable summary. Am. J. Clin. Nutr. 2011, 94, 313S-321S. [CrossRef]

19. Klee, G.G. Cobalamin and folate evaluation: Measurement of methylmalonic acid and homocysteine vs vitamin $\mathrm{B}_{12}$ and folate. Clin. Chem. 2000, 46, 1277-1283.

20. Carmel, R. Diagnosis and management of clinical and subclinical cobalamin deficiencies: Why controversies persist in the age of sensitive metabolic testing. Biochimie 2013, 95, 1047-1055. [CrossRef]

21. Bilici, A.; Sonkaya, A.; Ercan, S.; Ustaalioglu, B.B.; Seker, M.; Aliustaoglu, M.; Orcun, A.; Gumus, M. The changing of serum vitamin B12 and homocysteine levels after gastrectomy in patients with gastric cancer: Do they associate with clinicopathological factors? Tumour Biol. 2015, 36, 823-828. [CrossRef] [PubMed]

22. Hu, Y.; Kim, H.I.; Hyung, W.J.; Song, K.J.; Lee, J.H.; Kim, Y.M.; Noh, S.H. Vitamin $B_{12}$ deficiency after gastrectomy for gastric cancer: An analysis of clinical patterns and risk factors. Ann. Surg. 2013, 258, 970-975. [CrossRef] [PubMed]

23. Sakuta, H.; Suzuki, T.; Yasuda, H.; Wakiyama, H.; Hase, K. Plasma vitamin B12, folate and homocysteine levels in gastrectomized men. Clin. Nutr. 2005, 24, 244-249. [CrossRef] [PubMed]

24. Lim, C.H.; Kim, S.W.; Kim, W.C.; Kim, J.S.; Cho, Y.K.; Park, J.M.; Lee, I.S.; Choi, M.G.; Song, K.Y.; Jeon, H.M.; et al. Anemia after gastrectomy for early gastric cancer: Long-term follow-up observational study. World J. Gastroenterol. 2012, 18, 6114-6119. [CrossRef] [PubMed]

(C) 2019 by the authors. Licensee MDPI, Basel, Switzerland. This article is an open access article distributed under the terms and conditions of the Creative Commons Attribution (CC BY) license (http:/ / creativecommons.org/licenses/by/4.0/). 